EPJ Web of Conferences 41, 05043 (2013)

DOI: $10.1051 /$ epjconf/20134105043

(C) Owned by the authors, published by EDP Sciences, 2013

\title{
Molecular wave packet dynamics decelerated by solvent environment: A theoretical approach
}

\author{
S. Thallmair ${ }^{1,2}$, M. Kowalewski ${ }^{1,3}$, B. P. Fingerhut ${ }^{1,4}$, C. F. Sailer ${ }^{2}$, and R. de Vivie-Riedle ${ }^{1}$ \\ ${ }^{1}$ Department Chemie, LMU München, Butenandtstr. 11, D-81377 München, Germany \\ ${ }^{2}$ LS für BioMolekulare Optik, LMU München, Oettingenstr. 67, D-80538 München, Germany \\ ${ }^{3}$ present address: Department of Information Technology, Uppsala Universitet, SE-751 05 Uppsala, \\ Sweden \\ ${ }^{4}$ present address: Department of Chemistry, University of California, Irvine, California 92697-2025, \\ USA
}

\begin{abstract}
We present a new dynamic continuum ansatz to describe the frictional force exerted on moving wave packets in a solvent cage. The solvent interferes on the femtosecond time scale, but decides the reaction outcome.
\end{abstract}

\section{Photoinduced bond cleavage in solution}

Most reactions in organic as well as biochemistry take place in solution. Electrostatic solvent effects are known to change the reactivity of the solute dramatically. The same holds for photochemical reactions where often dissociative processes are included. Here not only the electrostatic influence but also the dynamic impact of the solvent becomes important. For bulky leaving groups, like in phosphonium salts [1], the solvent cage substantially decelerates the photogenerated fragments depending on the viscosity of the solvent. Subsequent distance dependent processes like electron or energy transfer between the photofragments become feasible as the fragments are kept at a reasonable distance. Also on the shorter time scale below $200 \mathrm{fs}$ frictional deceleration is essential. The molecular system gets the chance to deposit energy into motions additional to the primary dissociation coordinate. Thereby regions decisive for the branching into competing product channels can be reached.

\section{Experimental and quantum chemical evidence}

Photoinduced bond cleavage is often employed for the generation of carbocations and radicals. Possible precursors are phosphonium salts [1], for example diphenylmethyltriphenylphosphonium salt. After a local $\pi-\pi^{*}$ excitation on the photo-leaving group with a fs laser pulse $(275 \mathrm{~nm}, 35 \mathrm{fs}$ FWHM), the bond cleavage process takes place. Ultrafast broadband transient absorption measurements as well as nanosecond pump-probe experiments exclusively show the formation of diphenylmethyl cations [2].

High level quantum chemical calculations $(\operatorname{CASSCF}(10,10))$ are performed taking phenylmethylphenylphosphonium ion as model system. They confirm that the observed cations generated by photoinduced heterolysis are thermodynamic stable. Contrary to the experimental observations, theory predicts homolytic bond cleavage as the initial process. A localized conical

This is an Open Access article distributed under the terms of the Creative Commons Attribution License 2.0, which permits unrestricted use, distribution, and reproduction in any medium, provided the original work is properly cited. 
intersection (CoIn) coupling the homolytic and heterolytic product channel could in principle lead to a fast depletion of the radical population. To reach the CoIn the C-C-P angle as additional coordinate is needed (Fig. 1). Quantum dynamical calculations on a related system [3] showed that the CoIn is missed by the dissociating wave packet (WP).

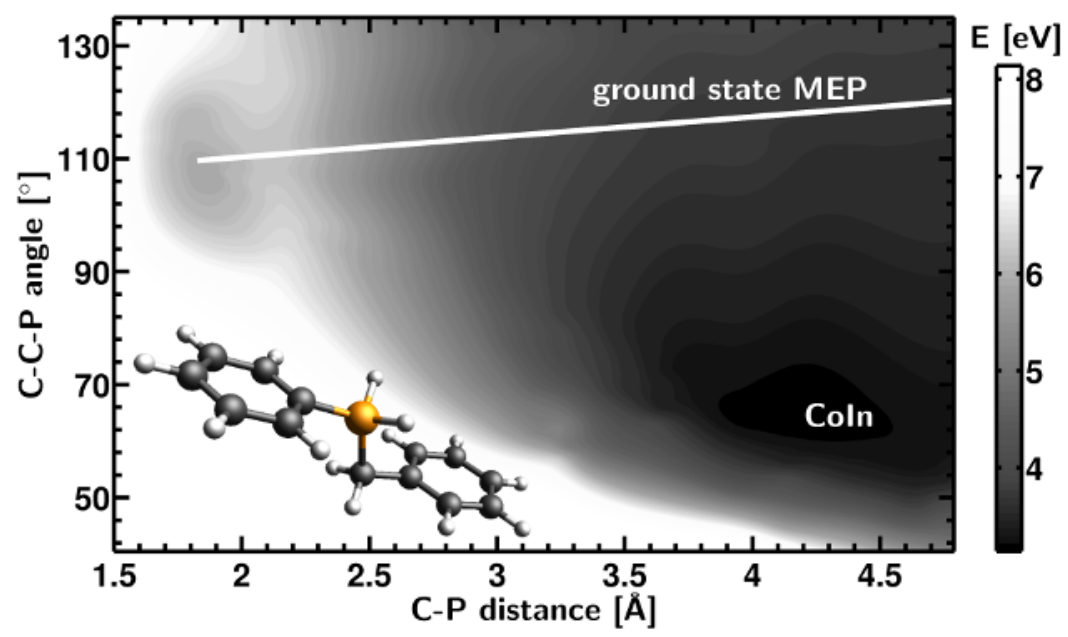

Fig. 1. Two dimensional potential energy surface of the first excited state of the phenylmethylphenylphosphonium ion (inlay). In addition to the primary dissociation coordinate, the C-P distance, the C-C-P angle is needed to reach the conical intersection (CoIn) which couples the homolytic and the heterolytic product channel. The black line indicates the direction of the minimum energy path (MEP) in the ground state.

\section{Dynamic continuum ansatz}

For the selected system both homolytic and heterolytic bond cleavage lead to the formation of one neutral and one positively charged fragment. Therefore the electrostatic solvent effect cannot change the reaction dynamics. Only the dynamic effect of the solvent comes into play. During the bond cleavage both fragments are accelerated. This leads to a deformation of the first solvation shell which in response slows down the acceleration process.

Our new dynamic continuum ansatz treats the frictional deceleration in the quantum mechanical formalism including the dynamic viscosity and Stokes' law. In every time step we decelerate the dissociating part of the WP in momentum space (k-space). The frictional force is represented by a decelerating shift $\Delta k_{W P}$ in the $\mathrm{k}$-space which is calculated via the expectation value of momentum $\left\langle k_{W P}\right\rangle$ of the WP:

$$
\Delta k_{\mathrm{WP}}=-6 \pi \eta R \frac{\left\langle k_{\mathrm{WP}}\right\rangle}{m} \Delta t
$$

where $\eta$ is the dynamic viscosity, $R$ the Onsager radius and $m$ the mass of the fragment. As a WP has a finite width, we have to differentiate between the part of the WP which already dissociates and the part which is still oscillating in the Franck-Condon region. Therefore we apply a sigmoid shape function, which is fitted to the cavity surface area along the reaction coordinate (Fig. 2, left). To ensure that only the dissociating part of the WP is influenced by the solvent environment, we employ the antiderivative of the sigmoid shape function $F_{\text {sig }}(x)$ to calculate the decelerated WP $\psi_{\text {dec }}(t)$ :

$$
\psi_{\mathrm{dec}}(t)=e^{i \Delta k_{\mathrm{WP}} F_{\mathrm{sig}}(x)} \psi(t) .
$$




\section{Results for the dynamic solvent effect}

Figure 2 (right) shows the deceleration of the WP in the first few hundred femtoseconds (solid line) compared to a propagation without the dynamic continuum ansatz (dashed line). The propagation starts at a C-P distance of $2.4 \AA$ behind the barrier in the first excited electronic state (Fig. 1). The WP is accelerated along the C-P coordinate due to the dissociative character of the potential. The bond separation up to a C-P distance of $3.5 \AA$ takes place very fast. But already at $3.0 \AA$ the friction becomes dominant and slows down the dissociation process. After $200 \mathrm{fs}$ the WP has lost most of its kinetic energy. The elongation of the C-P distance is dramatically slowed down around $4.0-4.5 \AA$. This is exactly the region of the CoIn (Fig. 1). As now the initial momentum of the WP in C-P direction is reduced, the WP can follow the gradient which points directly to the CoIn. Here the direct and ultrafast depletion of the radical population can take place.
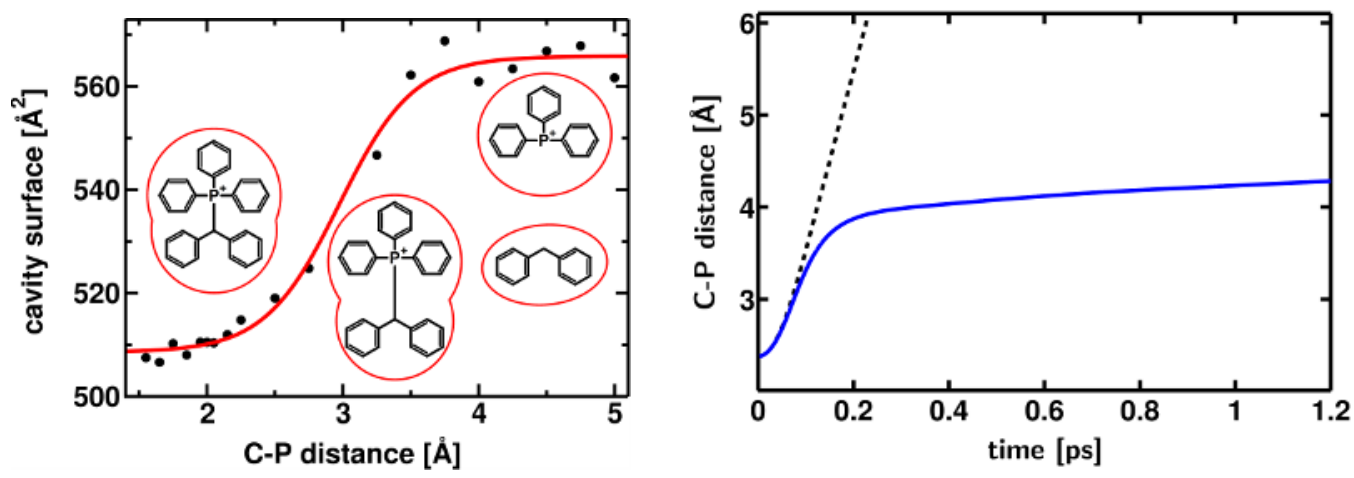

Fig. 2. Cavity surface area plotted against C-P distance (black dots, left) together with the fitted sigmoid shape function (solid line). Expectation value of the C-P distance during the bond cleavage process in the phenylmethyl-phenylphosphonium ion (right). The propagation was performed in one dimension along the minimum energy path in the ground state with (solid line) and without (dashed line) the dynamic continuum ansatz.

The photolytic bond cleavage in the phenylmethylphenylphoshonium ion is an outstanding example for the demonstration of the dynamic solvent effect. Due to the properties of the molecular system the electrostatic effect has no influence and can be neglected. The dynamic solvent effect treated with our new dynamic continuum ansatz has a distinct impact on the few hundred fs time scale and can explain the experimentally observed reaction outcome.

\section{References}

1. J. Ammer, H. Mayr, Macromolecules 43, 1719 (2010)

2. C. F. Sailer, R. B. Singh, J. Ammer, E. Riedle, I. Pugliesi, Chem. Phys. Lett. 512, 60 (2011)

3. B. P. Fingerhut, D. Geppert and R. de Vivie-Riedle, Chem. Phys. 343, 329 (2008) 